\title{
String no ring
}

\author{
Edward Y. Sako, MD, PhD
}

\footnotetext{
From the Department of Cardiothoracic Surgery, University of Texas Health Science Center at San Antonio, San Antonio, Tex.

Disclosures: E.Y.S. has a financial relationship with Medtronic, Inc.

Received for publication Sept 22, 2016; accepted for publication Sept 22, 2016; available ahead of print Nov 17 , 2016.

Address for reprints: Edward Y. Sako, MD, PhD, Department of Cardiothoracic Surgery, University of Texas Health Science Center at San Antonio, Mail Code 7841, 7703 Floyd Curl Dr, San Antonio, TX 78229-3900 (E-mail: sako@uthscsa.edu).

J Thorac Cardiovasc Surg 2017;153:278-9

$0022-5223 / \$ 36.00$

Copyright $(\underset{2}{ } 2016$ by The American Association for Thoracic Surgery

http://dx.doi.org/10.1016/j.jtcvs.2016.09.078
}

The challenge of how to address mitral valve regurgitation in the face of aortic valve disease persists. In particular, what to do when the regurgitation appears to be functional and therefore may improve with correction of the aortic valve pathology? Is the possible benefit of fixing the mitral valve worth the risk of increased ischemic and pump time?

In the accompanying article by Komeda, ${ }^{1}$ a method of dealing with the mitral valve but avoiding opening the left atrium, thereby reducing valuable ischemic time, is

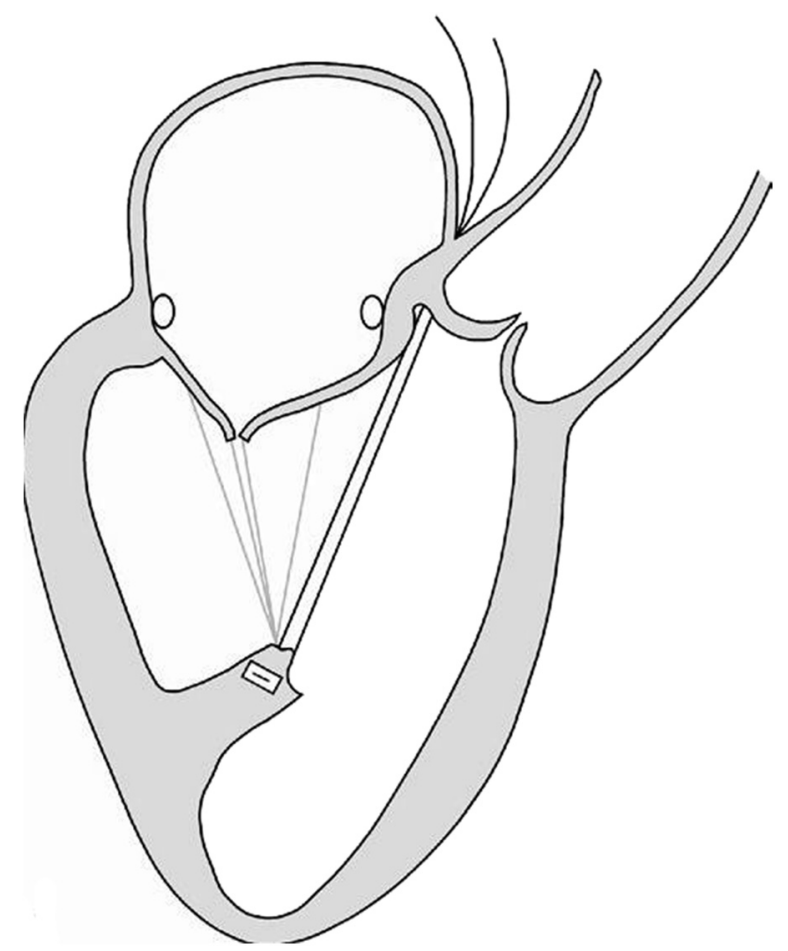

FIGURE 1. Ring plus string. Reprinted from Langer F, Schafers HJ. RING plus STRING: papillary muscle repositioning as an adjunctive repair technique for ischemic mitral regurgitation. J Thorac Cardiovasc Surg. 2007;133:247-9. ${ }^{3}$

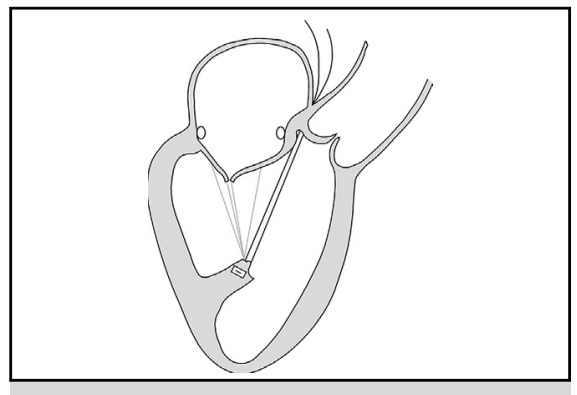

Ring plus string. Reprinted with permission.

Central Message

An expeditious option to treat concurrent mitral regurgitation at the time of aortic valve replacement is described.

See Article page 275

outlined. It is patterned in part after techniques previously described in which the tips of the papillary muscles are drawn up toward the annulus to reduce the tethering effect of ventricular dilation. ${ }^{2,3}$ In this particular version as in that described by Langer and Schafers, ${ }^{3}$ an added benefit is that the amount of pulling can be determined in the functioning heart under echocardiographic guidance. In the procedure by Langer and Schafers, ${ }^{3}$ the papillary muscle component was combined with an annuloplasty (ring plus string) (Figure 1).

In most cases of mitral regurgitation thought to be functional, that is secondary to some form of cardiomyopathy; 2 major mechanisms may be involved. The first is annular dilation, and the second is leaflet tethering. This particular technique addresses only the second mechanism. Therefore, it is clear that this method is limited and stresses the need to clearly elucidate the mechanism of regurgitation. In this series, 4 of the 18 patients who had significant mitral regurgitation associated with aortic valve disease underwent mitral annuloplasty, thereby obviating the advantage of avoiding an atriotomy.

In the end, it is useful as another item in one's "bag of tricks" to address the complex problems we face. Exactly when to use it and what the medium- to long-term efficacy remain to be seen. Perhaps a follow-up to this series is warranted. Nonetheless, an approach such as this is important to consider. There is the suggestion, although not conclusive, that mitral regurgitation may not improve without direct intervention even after the aortic pathology is treated. ${ }^{4}$ 


\section{References}

1. Komeda M. Quick but effective surgery for functional mitral regurgitation secondary to aortic valve disease. J Thorac Cardiovasc Surg. 2017;153: 275-7.

2. Kron IL, Green GR, Cope JT. Surgical relocation of the posterior papillary muscle in chronic ischemic mitral regurgitation. Ann Thorac Surg. 2002;74:600-1.

3. Langer F, Schafers HJ. RING plus STRING: papillary muscle repositioning as an adjunctive repair technique for ischemic mitral regurgitation. J Thorac Cardiovasc Surg. 2007;133:247-9.

4. Schubert SA, Yarboro LT, Madala S, Ayunipudi K, Kron IL, Kern JA, et al. Natural history of coexistent mitral regurgitation following aortic valve replacement. J Thorac Cardiovasc Surg. 2016;151:1032-42. 